\title{
Integration of inspiratory and expiratory intra- abdominal pressure: a novel concept looking at mean intra-abdominal pressure
}

\author{
Siavash Ahmadi-Noorbakhsh", Manu LNG Malbrain $2,3^{*}$
}

\begin{abstract}
Background: The intra-abdominal pressure (IAP) is an important clinical parameter that can significantly change during respiration. Currently, IAP is recorded at end-expiration (IAP ee), while continuous IAP changes during respiration ( $\triangle \mathrm{A} A \mathrm{P})$ are ignored. Herein, a novel concept of considering continuous IAP changes during respiration is presented.

Methods: Based on the geometric mean of the IAP waveform (MIAP), a mathematical model was developed for calculating respiratory-integrated MIAP (i.e. $\mathrm{MIAP}_{\mathrm{ri}}=\mathrm{IAP}_{\mathrm{ee}}+i \cdot \Delta \mathrm{IAP}$ ), where ' $i$ ' is the decimal fraction of the inspiratory time, and where $\triangle I A P$ can be calculated as the difference between the IAP at end-inspiration $\left(I A P_{\mathrm{e}}\right)$ minus $I A P_{\text {ee }}$. The effect of various parameters on IAP ee and MIAP ri was evaluated with a mathematical model and validated afterwards in six mechanically ventilated patients. The MIAP of the patients was also calculated using a CiMON monitor (Pulsion Medical Systems, Munich, Germany). Several other parameters were recorded and used for comparison.
\end{abstract}

Results: The human study confirmed the mathematical modelling, showing that MIAP $\mathrm{ri}_{\mathrm{i}}$ correlates well with MIAP $\left(R^{2}=0.99\right)$; $M I A P_{r i}$ was significantly higher than $\mathrm{IAP}_{\text {ee }}$ under all conditions that were used to examine the effects of changes in $I A P_{e e}$, the inspiratory/expiratory (I:E) ratio, and $\triangle \mathrm{AP}(P<0.001)$. Univariate Pearson regression analysis showed significant correlations between $\mathrm{MIAP}_{\mathrm{ri}}$ and IAP $\mathrm{IA}(R=0.99)$, IAP ee $(R=0.99)$, and $\triangle \mathrm{IAP}(R=0.78)(P<0.001)$; multivariate regression analysis confirmed that IAP ee (mainly affected by the level of positive end-expiratory pressure, PEEP), $\triangle I A P$, and the l:E ratio are independent variables $(P<0.001)$ determining MIAP. According to the results of a regression analysis, MIAP can also be calculated as

$$
\mathrm{MIAP}=-0.3+\mathrm{IAP}_{\mathrm{ee}}+0.4 \cdot \Delta \mathrm{IAP}+0.5 \cdot \frac{I}{E}
$$

Conclusions: We believe that the novel concept of MIAP is a better representation of IAP (especially in mechanically ventilated patients) because MIAP takes into account the IAP changes during respiration. The MIAP can be estimated by the MIAP $\mathrm{ri}_{\mathrm{i}}$ equation. Since $\mathrm{MIAP}_{\mathrm{ri}}$ is almost always greater than the classic IAP, this may have implications on end-organ function during intra-abdominal hypertension. Further clinical studies are necessary to evaluate the physiological effects of MIAP.

\section{Introduction}

The intra-abdominal pressure (IAP) is an important clinical parameter with major prognostic impact $[1,2]$. An unrecognised pathological increase in IAP eventually leads to intra-abdominal hypertension (IAH) and

\footnotetext{
* Correspondence: manu.malbrain@skynet.be

2Executive Committee, World Society of the Abdominal Compartment

Syndrome (WSACS), Dreef 1, Lovenjoel, 3360, Belgium

Full list of author information is available at the end of the article
}

abdominal compartment syndrome (ACS) [3,4], which result in significant morbidity and mortality [5]. Thus, recognition and reliable measurement of IAP are the first important steps for prevention and management of IAH and ACS in critically ill patients [6].

Assuming no respiratory movement, the IAP would be relatively constant and primarily determined by body posture and anthropomorphy (e.g. body mass index) [3,7].

\section{SpringerOpen ${ }^{\circ}$}

(C) 2012 Ahmadi-Noorbakhsh and Malbrain; licensee Springer This is an open access article distributed under the terms of the Creative Commons Attribution License (http://creativecommons.org/licenses/by/2.0), which permits unrestricted use, distribution, and reproduction in any medium, provided the original work is properly cited. 
The IAP may be affected by conditions influencing intraabdominal volume and abdominal compliance $\left(C_{\mathrm{ab}}\right)$ $[3,8,9]$. Further, the complex interaction between intraabdominal volume and $C_{\mathrm{ab}}$ during respiration (Figure 1) may significantly [10] and frequently (12 to 40 changes per minute) change the IAP (Figure 2), with more intense effects during positive-pressure mechanical ventilation or the presence of positive end-expiratory pressure (PEEP) [10-12].

According to the current consensus definitions of the World Society of the Abdominal Compartment Syndrome (WSACS), the IAP should be measured at end-expiration $\left(\operatorname{IAP}_{\text {ee }}\right)$ [13], referred to as the 'classic IAP' throughout the text. However, the IAP $_{\mathrm{ee}}$ is only a single component of an ever-changing trend and thus does not incorporate a considerable portion of this IAP trend (Figure 2). The objectives of this study were to develop and validate a novel IAP measurement concept to consider IAP changes during respiration and to identify independent variables influencing IAP within this novel concept.

\section{Methods}

\section{Part A: mathematical model}

A set of numerous IAP values occurs for a patient during a single respiratory cycle. The central tendency of a set of values can be calculated by the mathematical function of the 'mean'. In determining the mean IAP, the arithmetic mean for IAP $_{\text {ee }}$ and the end-inspiratory IAP (IAP $\left.{ }_{\mathrm{ei}}\right)$ was described previously [14], calculated by dividing the sum of the values by the number of values. However, employing the arithmetic mean for the IAP waveform is mathematically incorrect. Instead, the mean of a waveform can be calculated by the 'geometric mean' function. The geometric mean is calculated by dividing the area under the waveform in a definite interval (i.e. the definite integral of the waveform) by the value of the definite interval [15]. Therefore, the mean IAP (MIAP) for a sample IAP waveform between the times $\left(T_{0}\right)$ and $(T)$ in Figure 2 can be calculated as follows:

$$
\operatorname{MIAP}_{\mathrm{ri}}=\left(\frac{1}{T-T_{0}}\right) \cdot \int_{T_{0}}^{T} \operatorname{IAP}(t) d t,
$$

where 'MIAP ${ }_{\text {ri }}$ ' is the respiratory-integrated MIAP, ' $T-T_{0}$ ' is the time interval for a full respiratory cycle, and 'IAP $(t) d t$ ' is the IAP at each time point $(t)$. The result would be a time-weighted mean for the IAP waveform. This is closely analogous with the critically important cardiovascular concept of mean arterial blood pressure [16-18], which is the geometric mean of the arterial blood pressure waveform $[19,20]$. Equation 1 may be simplified as follows (see the addendum) [21]:

$$
\mathrm{MIAP}_{\mathrm{ri}}=\mathrm{IAP}_{\mathrm{ee}}+i \cdot \Delta \mathrm{IAP}
$$

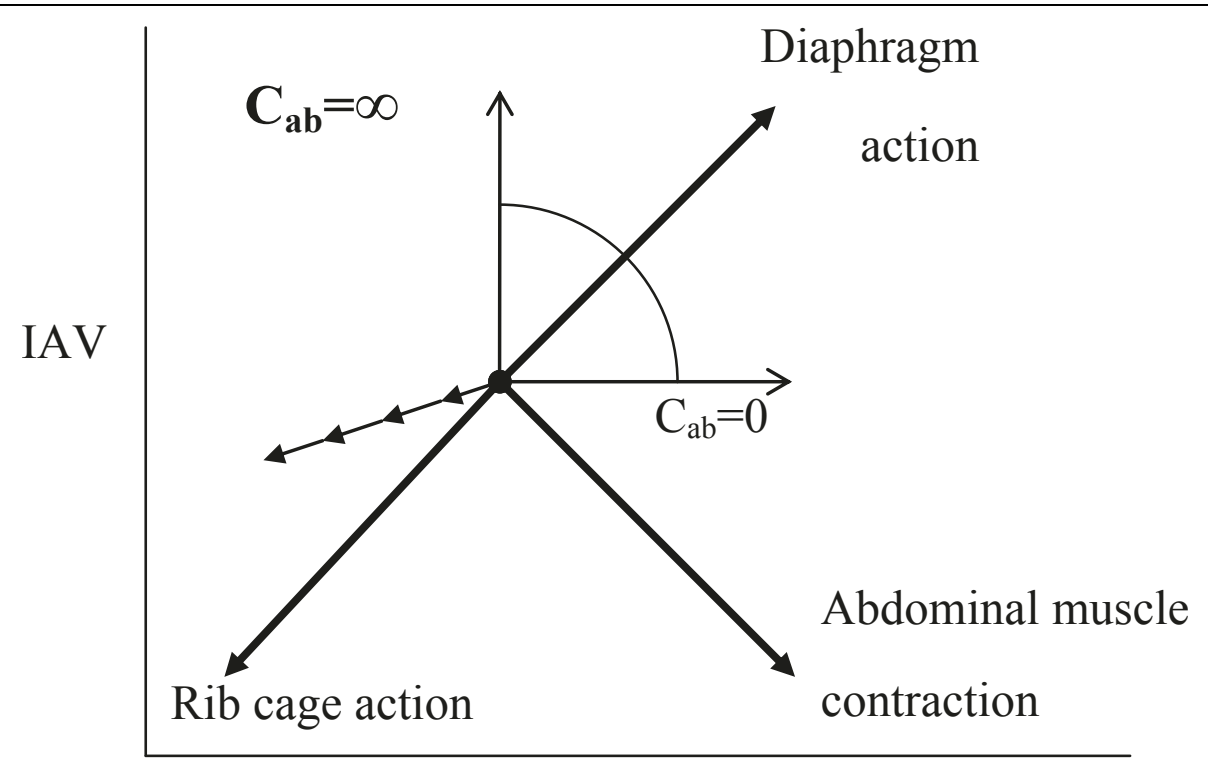

\section{IAP}

Figure 1 Relationship between intra-abdominal volume (IAV), abdominal wall compliance $\left(C_{\mathrm{ab}}\right)$ and intra-abdominal pressure (IAP) The directions of the movement of IAP on the $x$ axis and IAV on the $y$ axis associated with the isolated action of the rib cage inspiratory muscles, abdominal expiratory muscles, and the diaphragm are shown. The direction of the action of the diaphragm depends on the abdominal compliance. Adapted from de Keulenaer et al. [7]. 


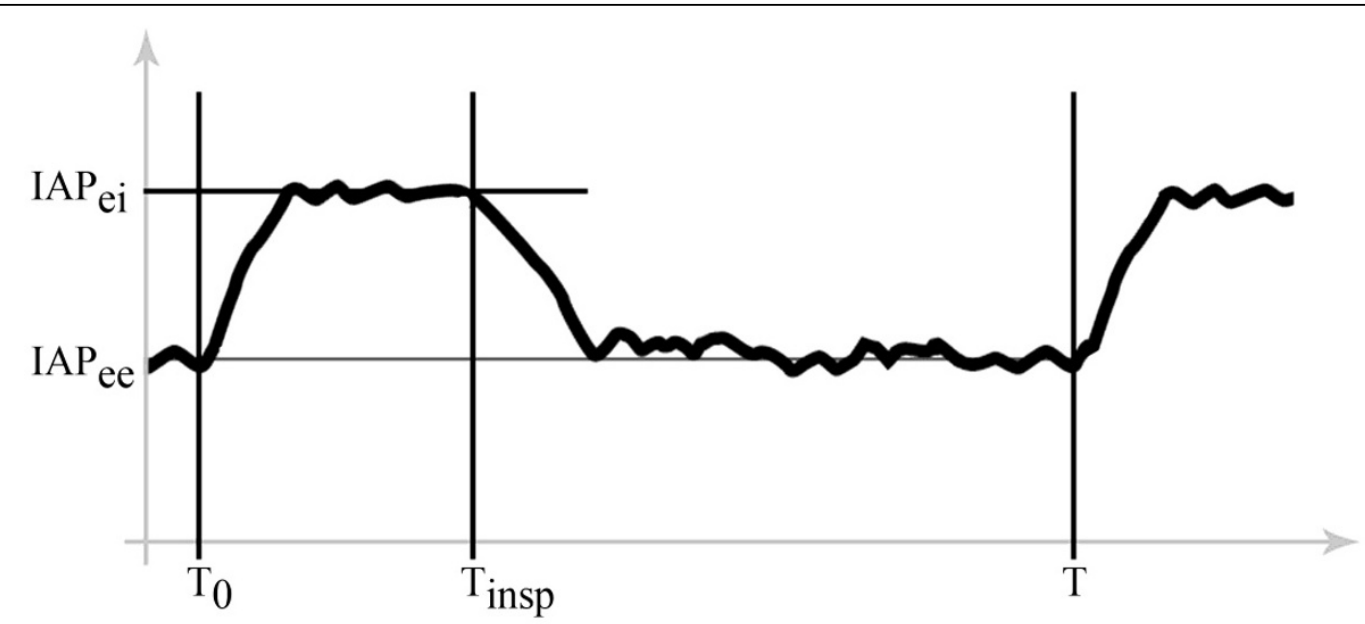

Time

Figure 2 Effect of respiration on intra-abdominal pressure (IAP). $T_{0}$, start of inspiration; $T_{\text {insp }}$ inspiratory time; $T$, total respiration time; IAP ee, end-expiratory IAP; IAP ei, end-inspiratory IAP.

where ' $i$ ' is the decimal fraction of the inspiratory time in a respiratory cycle and can be calculated from the inspiratory/expiratory $(I: E)$ ratio $(i=I /(I+E) ; 0<i<1)$ and $\Delta \mathrm{IAP}=\mathrm{IAP}_{\mathrm{ei}}-\mathrm{IAP}_{\mathrm{ee}}$. Since $\mathrm{IAP}_{\mathrm{ee}}, i$, and $\Delta \mathrm{IAP}$ can be assumed to be independent, a computerised iteration can be used for a set of values for each parameter to determine their effect on MIAP $_{r i}$ and to compare the MIAP ${ }_{\text {ri }}$ with the classic IAP.

The effects of IAP $_{\text {ee }}$ on MIAP ri $_{\text {and }}$ and the classic IAP were examined through a gradual increase of IAP $_{\mathrm{ee}}$ from 12 to $25 \mathrm{mmHg}$, with steps of $1 \mathrm{mmHg}$ (Figure 3). For each $\mathrm{IAP}_{\mathrm{ee}}$, a range of possible MIAP ri values was calculated according to Equation 2 with an I:E ratio of 4:1 and an $\triangle I A P$ of $8.16 \mathrm{mmHg}$ for the maximum MIAP $_{\text {ri }}$, and an $I: E$ ratio of $1: 4$ and an $\triangle \mathrm{IAP}$ of $1 \mathrm{mmHg}$ for the minimum MIAP ${ }_{\text {ri }}$. Because previous studies have shown a correlation between $\triangle \mathrm{IAP}$ and $\mathrm{IAP}_{\mathrm{ee}}$, the $\triangle \mathrm{IAP}$ was increased $10 \%$ for each $1 \mathrm{mmHg}$ increase in the $\mathrm{IAP}_{\mathrm{ee}}$.

The effects of the I:E ratio on MIAP $_{\mathrm{ri}}$ and the classic IAP were examined by a gradual increase in the $I: E$ ratio from 1:4 to $4: 1$ with steps of 0.5 (Figure 4). The amount of IAP $_{\text {ee }}$ was held constant $(19 \mathrm{mmHg})$. For each $I: E$ ratio, a range of possible MIAP $_{\text {ri }}$ values was calculated with an $\triangle \mathrm{IAP}$ of $7 \mathrm{mmHg}$ for the maximum $\mathrm{MIAP}_{\mathrm{ri}}$ and an $\triangle \mathrm{IAP}$ of $2 \mathrm{mmHg}$ for the minimum MIAP $\mathrm{P}_{\text {ri }}$.

The effects of $\triangle \mathrm{IAP}$ on MIAP $\mathrm{ri}_{\mathrm{i}}$ and the classic IAP were examined by a gradual increase in $\triangle \mathrm{IAP}$ from 1 to 5 $\mathrm{mmHg}$, with steps of $0.5 \mathrm{mmHg}$ (Figure 5). The amount of IAP ee was held constant $(19 \mathrm{mmHg})$. For each $\triangle \mathrm{IAP}$, a range of possible MIAP $_{\text {ri }}$ values was calculated with an I:E ratio of 4:1 for the maximum MIAP $_{\text {ri }}$ and an I:E ratio of 1:4 for the minimum MIAP ${ }_{\text {ri }}$.
Each of the abovementioned data sets was assumed to be a unique case, and the values shown in Figures 3,4,5 should not be considered as a trend in changes that can be obtained in a single patient.

\section{Part B: human pilot study}

In six ICU patients that were mechanically ventilated with Evita XL ventilators (Draeger, Lubeck, Germany), the mean IAP was automatically calculated as the geometrical mean (MIAP) via a balloon-tipped nasogastric tube connected to a CiMON monitor (Pulsion Medical Systems, Munich, Germany). The MIAP ri was also calculated according to Equation 2. Data were collected on respiratory settings, plateau and mean alveolar pressures $\left(P_{\text {plat }}, P_{\text {mean }}\right)$, PEEP, and dynamic compliance (calculated as the tidal volume (TV) divided by $\left(P_{\text {plat }}-\right.$ PEEP)). The $C_{\text {ab }}$ was calculated as TV divided by $\triangle \mathrm{IAP}$. The thoraco-abdominal index of transmission (TAI) was calculated as $\Delta P_{\text {alv }}\left(=P_{\text {plat }}-\right.$ PEEP) divided by $\triangle \mathrm{IAP}$, in which $P_{\mathrm{alv}}$ is the alveolar pressure.

The effects of IAP $_{\mathrm{ee}}$ on MIAP ${ }_{\mathrm{ri}}$ were examined by a gradual increase in PEEP from 0 to $15 \mathrm{cmH}_{2} \mathrm{O}$, with steps of $5 \mathrm{cmH}_{2} \mathrm{O}$ during a best-PEEP manoeuvre (20 measurements at each PEEP level in five patients, resulting in 80 measurements). The effects of $\triangle \mathrm{IAP}$ on MIAP ${ }_{\text {ri }}$ were examined by a gradual increase in TV from 250 to 1,000 $\mathrm{ml}$, with steps of $250 \mathrm{ml}$ during a low-flow pressurevolume loop (20 measurements at each TV level in five patients, resulting in 80 measurements). The effects of $I: E$ ratio on MIAP $_{\text {ri }}$ were examined by a gradual increase in the $I: E$ ratio from $1: 2$ to $2: 1$, with steps of 0.5 during a recruitment manoeuvre ( 9 measurements at each $I: E$ ratio in one patient, resulting in 45 measurements). 


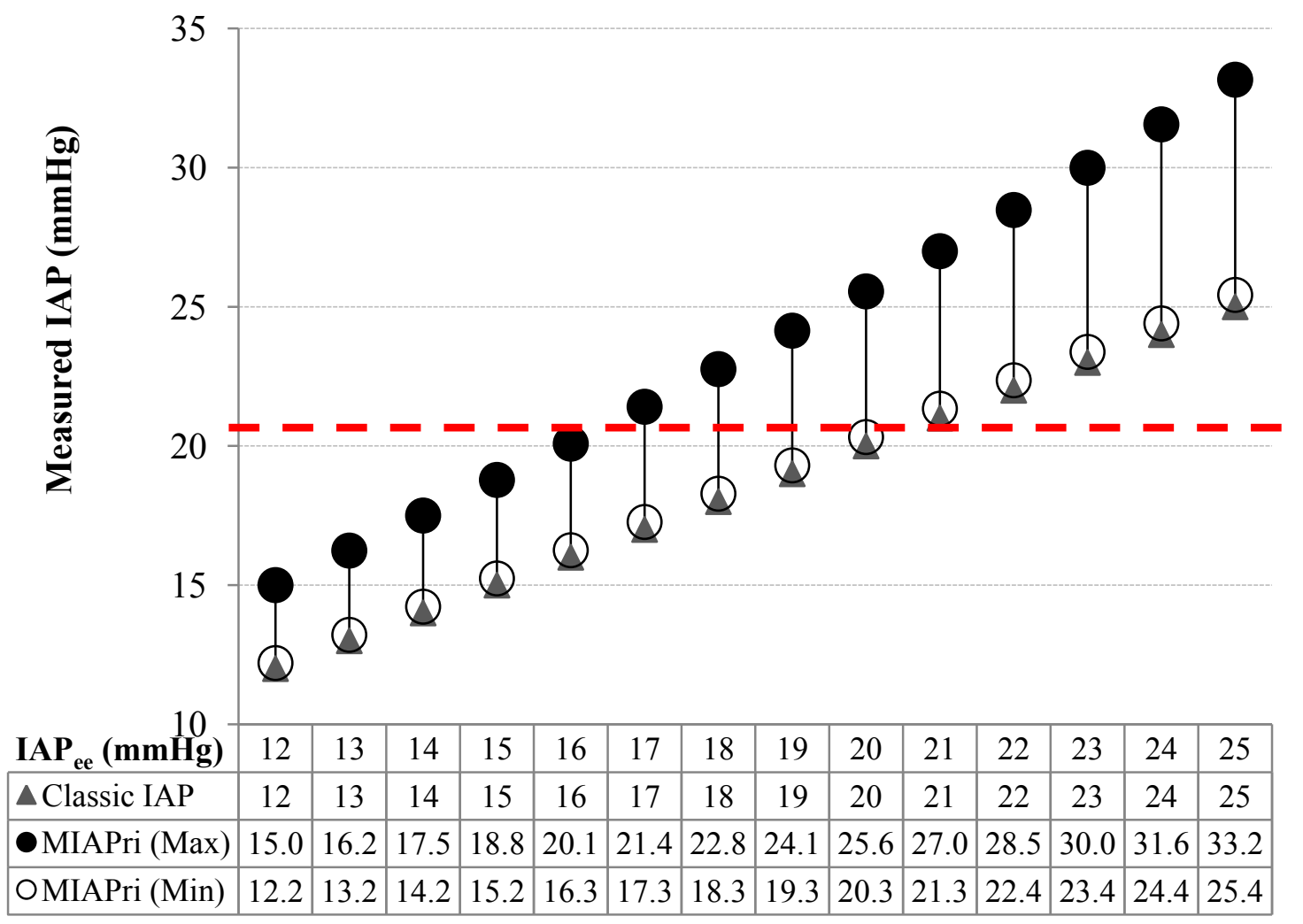

Figure 3 Mathematical modelling of IAP measurements for various end-expiratory IAP values (IAP ee). The classic (IAP ee) and novel $\left(M_{I A P_{r i}}\right)$ methods were used to measure the IAP. The dashed line represents the ACS threshold. The lines connecting the Max and Min MIAP ri values represent the range of possible MIAP ri values.

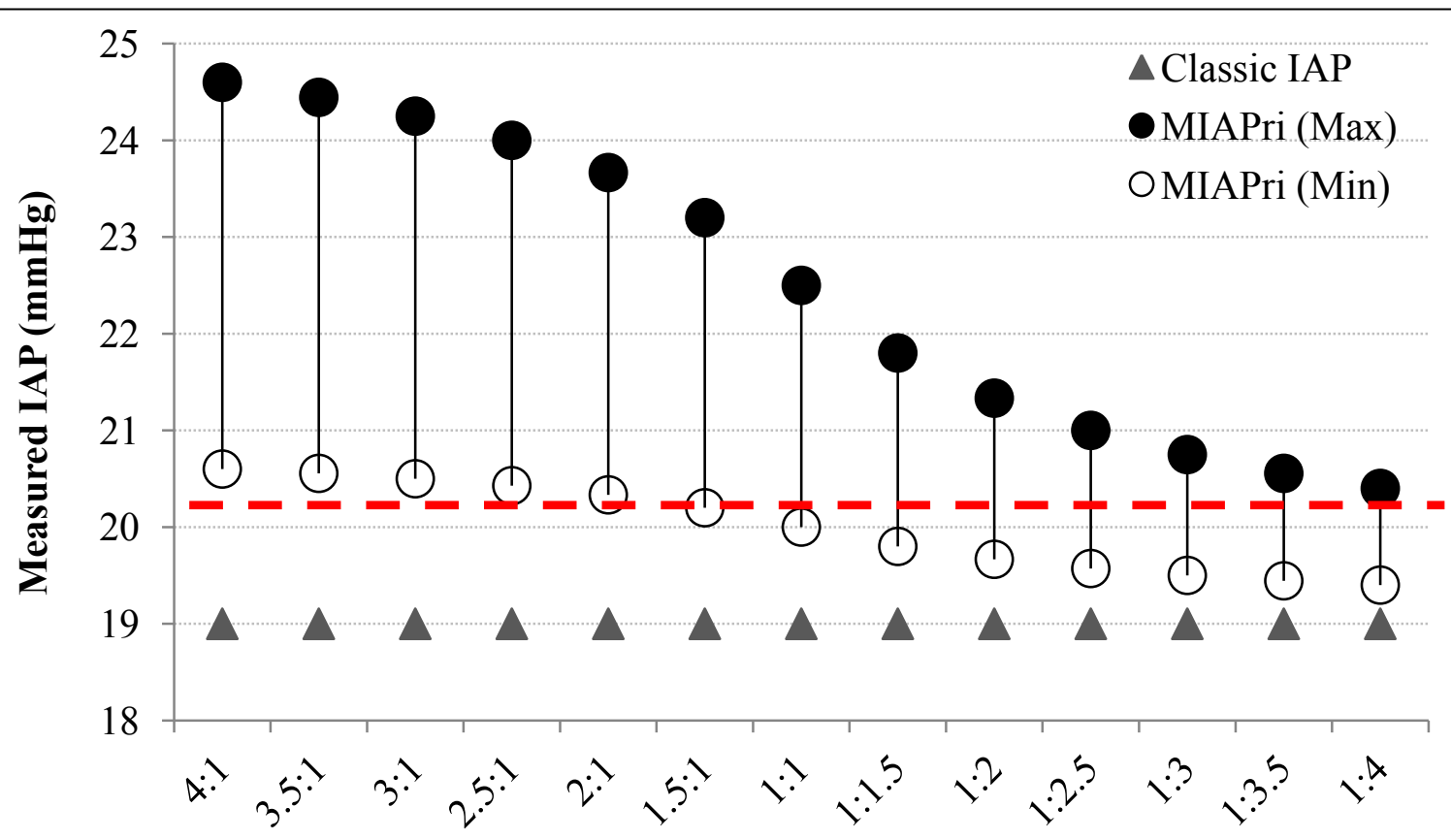

I:E Ratio

Figure 4 Mathematical modelling of IAP measurements for a constant $19 \mathrm{mmHg}$ end-expiratory IAP (IAP ee) and various I:E ratios. The $M_{M A P_{r i}}$ values were calculated for various I:E ratios. The classic (IAP $\left.\mathrm{P}_{\mathrm{ee}}\right)$ and novel $\left(\mathrm{MIAP}_{\mathrm{ri}}\right)$ methods were compared. For each l:E ratio, a range of possible MIAP ri values was calculated according to various $\triangle I A P$ values. The dashed line represents the ACS threshold. 


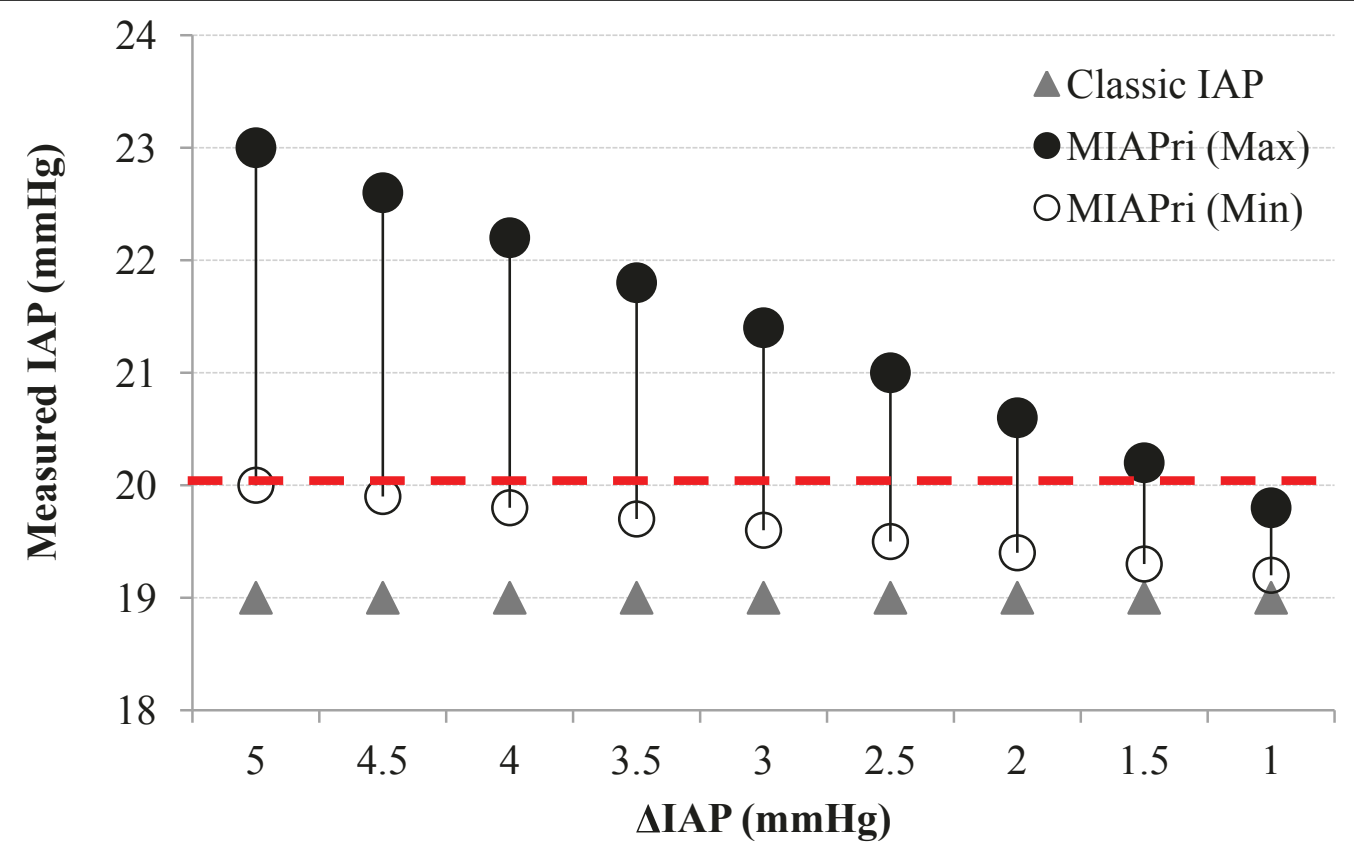

Figure 5 Mathematical modelling of IAP measurement for a constant $19 \mathrm{mmHg}$ end-expiratory IAP (IAP ee) and various $\triangle$ IAP. The classic $\left(I_{\mathrm{AP}} \mathrm{ee}\right)$ and novel $\left(\mathrm{MIAP}_{\mathrm{ri}}\right)$ methods were used to measure the IAP. The MIAP ri values were calculated for each $\triangle I A P$. A range of possible $M_{I A P_{r i}}$ values for each $\triangle I A P$ was calculated according to various I:E ratios. The dashed line represents the ACS threshold.

Statistical analysis was performed using SPSS software. Pearson correlation analysis and Bland and Altman analysis were performed. For comparisons between MIAP $_{\text {ri }}$ and $\mathrm{IAP}_{\text {ee }}$ at different levels of $\mathrm{IAP}_{\text {ee }}(\mathrm{PEEP}), \mathrm{TV}$, and $I$ : $E$ ratio, a two-tailed paired Student's $t$-test was performed. Data are expressed as the mean with the standard deviation (SD), unless specified otherwise. A $P$ value below 0.05 was considered statistically significant. The local EC and IRB approved the study, and informed consent was obtained from next of kin.

\section{Results}

\section{Part A: mathematical modelling}

According to Equation 2, three major independent parameters determine the $\mathrm{MIAP}_{\mathrm{ri}}$ : $\mathrm{IAP}_{\mathrm{ee}}, I: E$ ratio, and $\triangle I A P$. Therefore, for each IAP $_{\text {ee }}$, the MIAP ri depends on two other factors (Figure 3). For IAP $_{\text {ee }}$ values between 16 and $20 \mathrm{mmHg}$, the classic IAP remained below the ACS threshold (dashed line in Figure 3); however, the MIAP ri $_{\text {was }}$ able to exceed the ACS threshold. Furthermore, as seen in Figures 4 and 5, the classic IAP was continuously below the ACS threshold, but different ranges of probable MIAP $_{\text {ri }}$ values were above the ACS threshold. By changing the $I: E$ ratio, the MIAP $_{\text {ri }}$ values changed with dissimilar intensities (e.g. when the $I: E$ ratio decreased from $4: 1$ to $3.5: 1$, the intensity of changes in the MIAP $_{\text {ri }}$ values was less than that when the $I: E$ ratio decreased from $1.5: 1$ to $1: 1$;
Figure 4). Furthermore, for a constant $\operatorname{IAP}_{\mathrm{ee}}$, higher values for either the I:E ratio or $\triangle I A P$ were found to be capable of causing a wider range of possible MIAP $_{\text {ri }}$ values (Figures 4 and 5). Mathematically, for all instances in which the $\triangle \mathrm{IAP}$ was greater than 0 mmHg, the MIAP $_{\text {ri }}$ was larger than the classic IAP (see the addendum) [21].

\section{Part B: human pilot study}

Six mechanically ventilated patients (three severely burned patients and three surgical ICU patients) were studied. The male-to-female ratio was 2:1. Table 1 summarises the baseline patient demographics.

\section{Regression analysis and Bland and Altman analysis}

In total, 205 paired MIAP and MIAP $_{\text {ri }}$ measurements were performed with an identical statistical mean of $12.2 \pm 3.8$ mmHg. Figure 6A shows an excellent correlation between the MIAP and MIAP ri $\left(R^{2}=0.99, P<0.001\right)$. Analysis according to Bland and Altman showed a bias and precision of 0 and $0.2 \mathrm{mmHg}$, respectively, with small limits of agreement ranging from -0.4 to $0.5 \mathrm{mmHg}$ (Figure 6B). The percentage error was $3.5 \%$.

\section{Effect of IAP ee, I:E ratio, and $\triangle I A P$ on $M I A P_{r i}$}

Gradually increasing PEEP from 0 to $15 \mathrm{cmH}_{2} \mathrm{O}$ resulted in an increase in $\mathrm{MIAP}_{\text {ri }}$ from $11.7 \pm 4.1$ to $13.1 \pm$ $4.2 \mathrm{mmHg}(P<0.001)$. Meanwhile, $\mathrm{IAP}_{\mathrm{ee}}$ increased from $9.9 \pm 3.4$ to $11.9 \pm 3.7 \mathrm{mmHg}(P<0.001)$. Moreover, a gradual increase in the $I: E$ ratio from $0.5(1: 2)$ to $2(2: 1)$ 
Table 1 Patient characteristics at baseline

\begin{tabular}{lc}
\hline Parameter & Mean \pm SD \\
\hline Age & $59.5 \pm 14.4$ \\
SAPS-II & $43.5 \pm 11.6$ \\
APACHE-II & $21.8 \pm 8.6$ \\
SOFA & $9.5 \pm 4$ \\
BMI $\left(\mathrm{kg} / \mathrm{m}^{2}\right)$ & $28.6 \pm 4.7$ \\
IAP $_{\text {ei }}(\mathrm{mmHg})$ & $15.3 \pm 3.7$ \\
IAP ee $(\mathrm{mmHg})$ & $11.1 \pm 2.8$ \\
$\triangle I A P(\mathrm{mmHg})$ & $4.3 \pm 1.3$ \\
MIAP $(\mathrm{mmHg})$ & $12.9 \pm 3$ \\
IBP $(\mathrm{mmHg})$ & $12 \pm 3$ \\
TV $(\mathrm{ml})$ & $608 \pm 117$ \\
TV $(\mathrm{ml} / \mathrm{kg})$ & $7.2 \pm 1.2$ \\
RR $(/ \mathrm{min})$ & $17.7 \pm 2.1$ \\
$P_{\text {plat }}\left(\mathrm{cmH} \mathrm{m}_{2} \mathrm{O}\right)$ & $28 \pm 4.1$ \\
PEEP $\left(\mathrm{cmH}_{2} \mathrm{O}\right)$ & $9.2 \pm 3.3$
\end{tabular}

SAPS, simplified acute physiology score; APACHE, acute physiology and chronic health evaluation; SOFA, sequential organ failure assessment; BMI, body mass index; IAP ${ }_{\mathrm{ei}}$, end-inspiratory IAP; $I A P_{\mathrm{ee}}$, end-expiratory IAP; MIAP, mean IAP; IBP, intra-bladder pressure; TV, tidal volume; RR, respiratory rate;

$\mathrm{P}_{\text {plat, }}$ plateau airway pressure; $\mathrm{PEEP}$, positive end-expiratory pressure.

caused an increase in MIAP $_{\text {ri }}$ from $10.8 \pm 2.6$ to $12.9 \pm$ $2.9 \mathrm{mmHg}(P<0.001)$, while $\mathrm{IAP}_{\mathrm{ee}}$ increased from $9.7 \pm$ 2.3 to $10.4 \pm 2.5 \mathrm{mmHg}(P<0.001)$. In addition, gradually increasing TV from 250 to $1,000 \mathrm{ml}$ led to an increase in $\triangle \mathrm{IAP}$ from $2.1 \pm 1.1$ to $5.7 \pm 2.3(P<0.001)$. This increase in $\triangle$ IAP resulted in an increase in $\mathrm{MIAP}_{\text {ri }}$ from $11.6 \pm 4$ to $13.1 \pm 4.3 \mathrm{mmHg}(P<0.001)$, while IAP $_{\text {ee }}$ increased from $10.7 \pm 3.6$ to $10.9 \pm 3.5 \mathrm{mmHg}(P=\mathrm{NS})$. The MIAP was significantly higher than $\mathrm{IAP}_{\mathrm{ee}}$ at each PEEP level, $I: E$ ratio, and TV (Figure 7A,B,C; $P<0.001$ ).

The classic IAP of patients was below the IAH grade I threshold; however, the $\mathrm{MIAP}_{\text {ri }}$ significantly exceeded the threshold in several instances $(P<0.001$; Figure 7$)$.

\section{Univariate analysis}

Univariate Pearson regression analysis showed significant correlations between $\operatorname{MIAP}_{\mathrm{ri}}$ and $\operatorname{IAP}_{\mathrm{ei}}(R=0.99)$, $\mathrm{IAP}_{\mathrm{ee}}(R=0.99), \Delta \mathrm{IAP}(R=0.78)$, and $C_{\mathrm{ab}}(R=-0.74)$; between $\operatorname{IAP}_{\mathrm{ei}}$ and $\mathrm{IAP}_{\mathrm{ee}}(R=0.96), \Delta \mathrm{IAP}(R=0.86)$, and $C_{\mathrm{ab}}(R=-0.73)$; between $\mathrm{IAP}_{\mathrm{ee}}$ and $\triangle \mathrm{IAP}(R=0.7)$ and $C_{\mathrm{ab}}(R=-0.73)$; between $\Delta \mathrm{IAP}$ and $\Delta P_{\mathrm{alv}}(R=0.79)$ and $C_{\mathrm{ab}}(R=-0.58)$; and finally between TAI and $C_{\mathrm{ab}}$ $(R=-0.8)(P<0.001)$. Figure $8 \mathrm{~A}, \mathrm{~B}, \mathrm{C}$ shows some regression plots.

\section{Multivariate regression analysis}

Analyses showed that the IAP $_{\text {ee }}$ (mainly affected by PEEP), $\triangle \mathrm{IAP}$, and $I: E$ ratio were independent variables defining the MIAP (Table 2). According to the regression analysis in our sample population, the MIAP can also be calculated from the following simplified formula $(P<0.001)$, in which ' $I$ ' and ' $E$ ' are elements of the $I: E$ ratio:

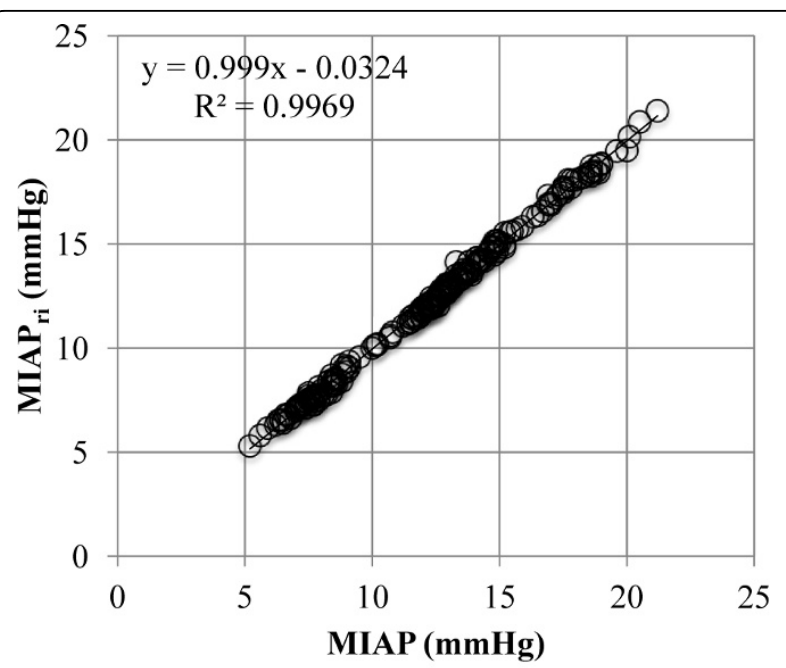

(a)

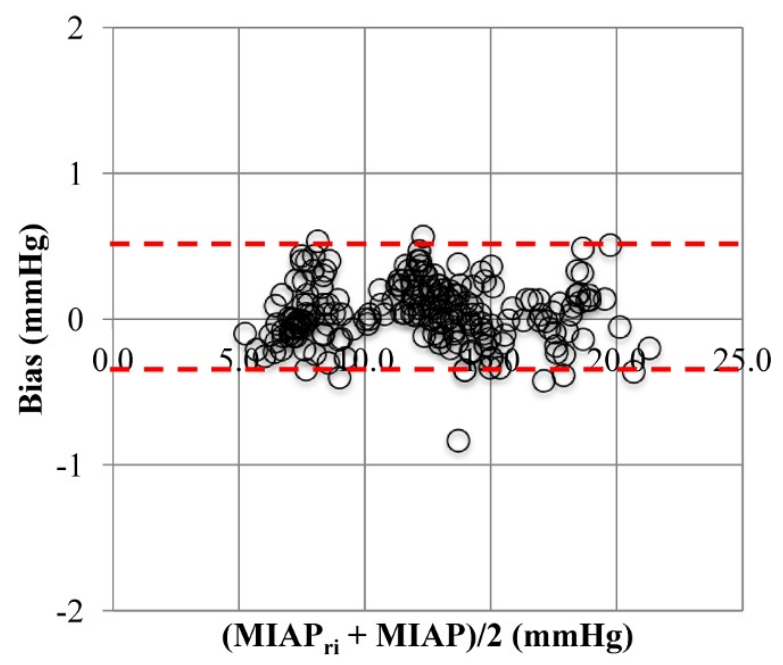

(b)

Figure 6 Regression plot and Bland and Altman analysis. (A) Regression plot comparing mean IAP measured via the geometric mean (MIAP) versus the respiratory-integrated MIAP (MIAP ri). There is an excellent correlation between the two methods. (B) Bland and Altman analysis comparing MIAP with MIAPri. The dashed red lines show the upper and lower limits of agreements.

$$
\mathrm{MIAP}_{\mathrm{ri}}=-0.3+\mathrm{IAP}_{\mathrm{ee}}+0.4 \cdot \Delta \mathrm{IAP}+0.5 \cdot \frac{I}{E}
$$

\section{Discussion}

A novel concept of IAP measurement based on the geometric mean of the IAP waveform was presented. The 


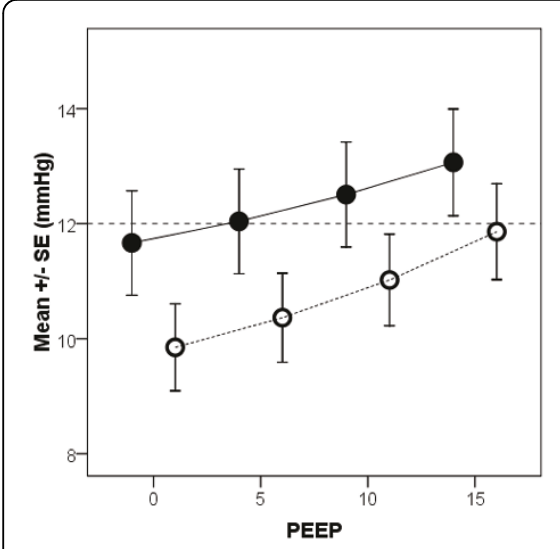

(a)

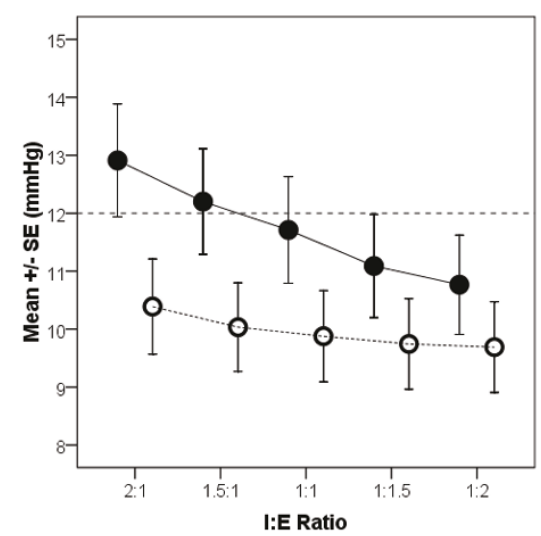

(b)

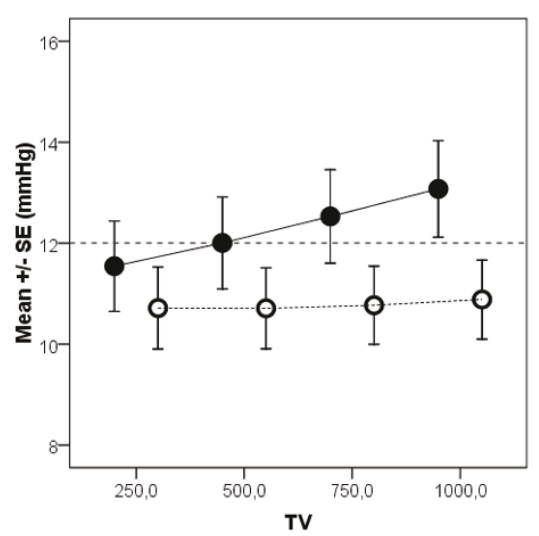

(c)

Figure 7 The effects of gradual increase of PEEP, I:E ratio, and TV. (A) The effect of gradual increase of PEEP on classic IAP (open circles) and the respiratory-integrated MIAP (MIAPri; closed circles). Both the classic IAP and MIAPri were increased significantly $(P<0.001)$. The MIAPri was significantly higher than the classic IAP for all PEEP levels $(P<0.001)$. The dashed line shows the $12 \mathrm{mmHg}$ IAH grade I threshold. (B) The effect of gradual increase of l:E ratio on IAP ee (open circles) and MIAP $P_{\text {ri }}$ (closed circles). Both the IAP ee and MIAP $P_{r i}$ were increased significantly $(P$ $<0.001)$. The MIAP ri was significantly higher than IAP ee for all l:E ratios $(P<0.001)$. The dashed line represents the $12 \mathrm{mmHg} I \mathrm{AH}$ grade I threshold.

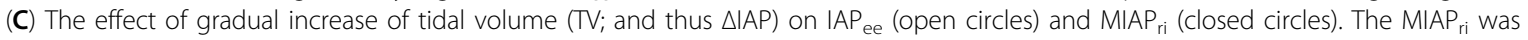
significantly higher than IAP ee at all TV values $(P<0.001)$. The dashed line shows the $12 \mathrm{mmHg} I \mathrm{AH}$ grade I threshold.

independent parameters determining the IAP in this concept were defined. The human pilot study validated the mathematical modelling with an excellent correlation. A significant difference was observed between the classic

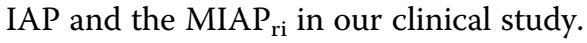

The human study confirmed that MIAP $_{\text {ri }}$ is as accurate as an automated geometric MIAP calculation by a CiMON monitor. More importantly, the higher the MIAP or IAP $_{\mathrm{ee}}$, the higher the $\triangle \mathrm{IAP}$ since $\triangle \mathrm{IAP}$ acts as an indirect marker of $C_{\mathrm{ab}}$. The $\triangle \mathrm{IAP}$ is correlated with $\Delta P_{\text {alv }}$ or is thus inversely correlated with dynamic compliance. As well, the higher the $C_{\mathrm{ab}}$, the lower the TAI. The human study confirmed the predictions of the mathematical modelling in which IAP $_{\text {ee }}$ (affected by different PEEP settings), $\triangle \mathrm{IAP}$, and $I: E$ ratio were recognised as the major independent determinants of MIAP ${ }_{\text {ri }}$. We also showed that in patients with IAH and under mechanical ventilation, the IAP may be influenced by ventilator settings.

The critical difference between the MIAP ${ }_{\text {ri }}$ and the classic IAP near the ACS threshold in our mathematical modelling, as well as the significantly higher MIAP $_{\text {ri }}$ than

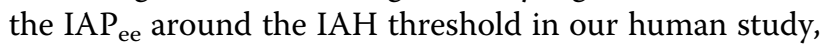
calls for future studies. The dissimilar intensity in MIAP $_{\text {ri }}$ changes under changes in the I:E ratio in Figure 4 may implicate the existence of critical points in the $I: E$ ratio, wherein changing this ratio may cause a more intense change in the MIAP $_{\text {ri }}$. Furthermore, since MIAP ri seems to be almost always larger than the classic IAP, relying only on the classic IAP may place some patients at risk of silent IAH or ACS. Although the aim of the current study was not to address these implications clinically, these findings indicate that further investigations should be performed on respiratory manoeuvres to manage IAH in mechanically ventilated patients (e.g. decreasing the $I$ : $E$ ratio and/or the $\triangle \mathrm{IAP}$, or maintaining the $I: E$ ratio in a predefined range).

A limitation of our study was the lack of data to evaluate the physiological difference between the MIAP $_{\text {ri }}$ and the classic IAP. However, this study only aimed to prove the concept and to set the stage for further studies. Therefore, we believe that the lack of physiological data does not limit our findings. Nonetheless, further studies on the clinical effects of this concept are necessary before it can be introduced in clinical practice.

\section{Conclusions}

A novel concept MIAP ri $_{\text {was }}$ presented to consider the IAP changes during respiration and was based on the geometric mean (MIAP) of the IAP waveform. An excellent correlation was observed between the results of the mathematical modelling and those obtained in real patients. Substantial differences were observed between the two IAP methods (the classic IAP measured at end expiration and the novel MIAP). Based on our findings, we believe that the novel concept of MIAP $_{\text {ri }}$ may be a better representation for the pressure concealed within the abdominal cavity. Further clinical studies are necessary to reveal the physiological effects of this novel concept. 


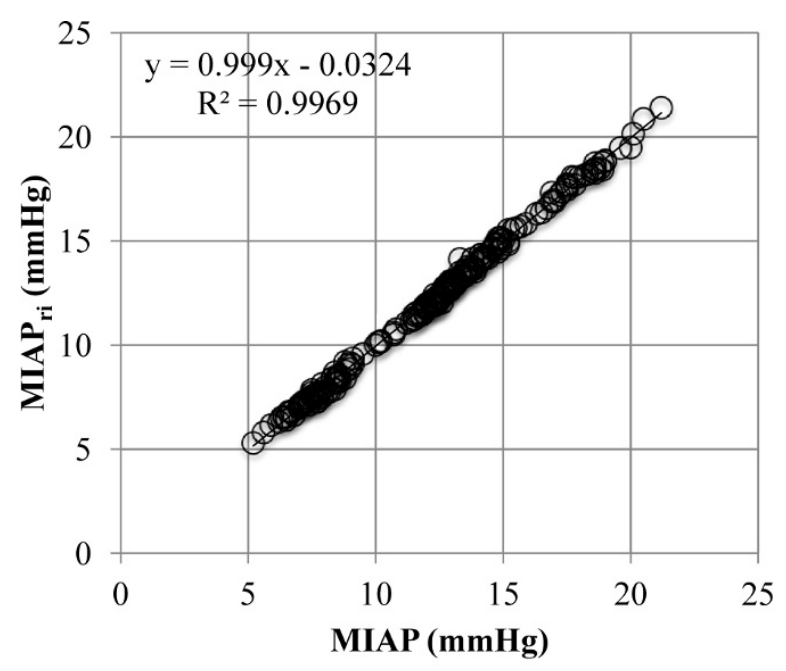

(a)

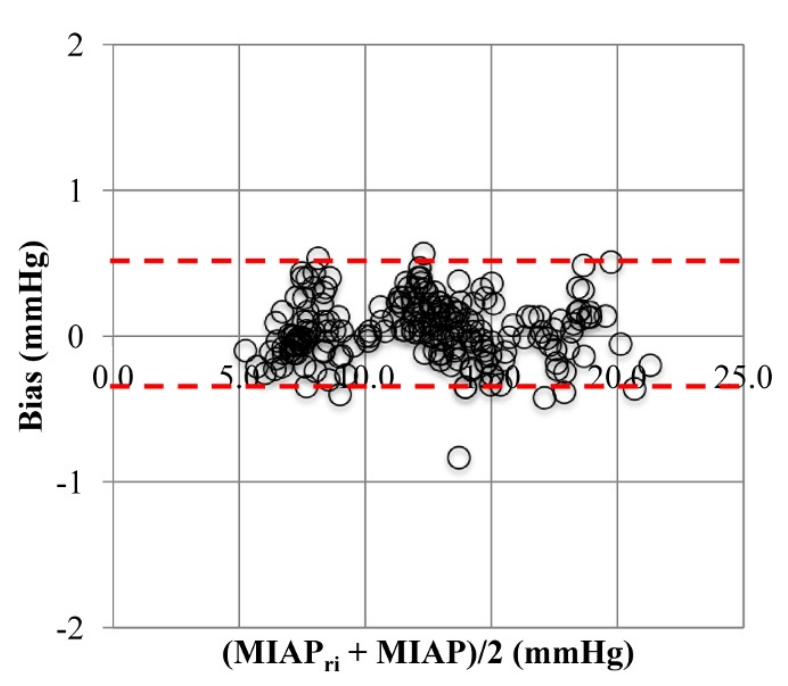

(b)

Figure 8 Linear regression plots. (A) Linear regression plot showing the respiratory-integrated mean intra-abdominal pressure (MIAP $\left.{ }_{\mathrm{ri}}\right)$ in relation to $\triangle I A P\left(=I A P_{e i}-I A P_{e e}\right.$, where IAP $P_{\mathrm{ei}}$ is the end-inspiratory IAP and IAP ee is the end-expiratory IAP). (B) Linear regression plot showing the respiratory changes of intra-abdominal pressure $(\triangle I A P)$ in relation to $\Delta P_{\text {alv }}\left(=P_{\text {plat }}\right.$ - PEEP, where $P_{\text {alv }}$ is the alveolar pressure, $P_{\text {plat }}$ is the plateau alveolar pressure, and PEEP is the positive end-expiratory pressure). (C) Linear regression plot showing the relation between the thoracoabdominal index of transmission (i.e. $T A I=\Delta P_{\text {alv }} / \Delta I A P$ ) and the abdominal wall compliance (i.e. $C_{a b}=T V / \Delta I A P$ ). 
Table 2 Multiple regression analysis looking for independent variables influencing MIAP

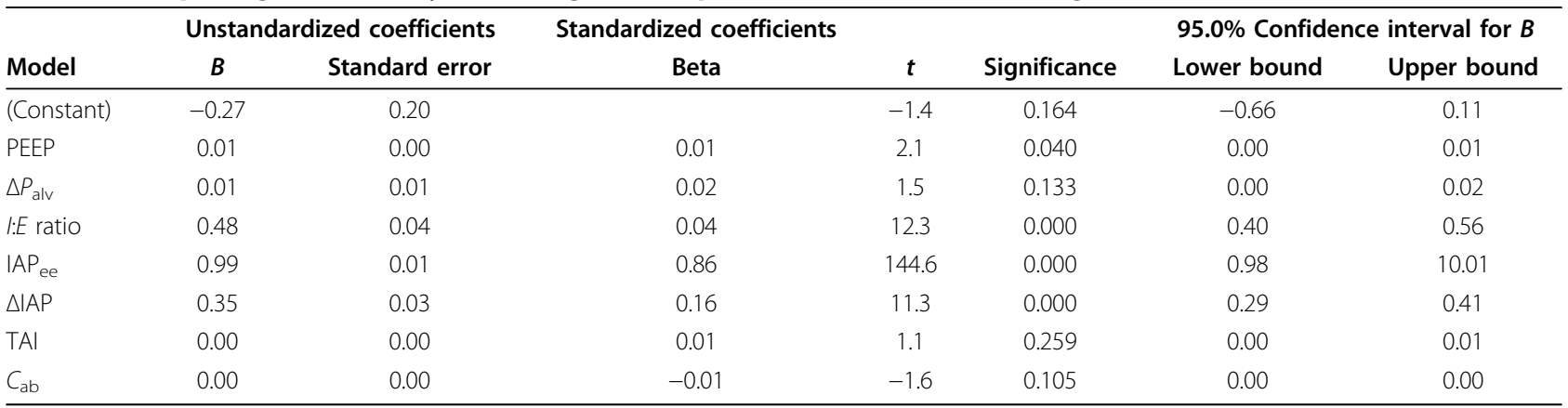

PEEP, positive end-expiratory pressure; $P_{\text {alv, }}$ alveolar pressure; I:E ratio, inspiratory-to-expiratory ratio; IAP ee, end-expiratory IAP; TAI, thoraco-abdominal index of transmission; $C_{a b}$, abdominal wall compliance.

\section{Addendum}

See additional file 1.

\section{Additional material}

Additional file 1: Mathematical model for calculation of mean intraabdominal pressure, taking into account integration of inspiratory and expiratory intra-abdominal pressure.

\begin{abstract}
Abbreviations
ACS: abdominal compartment syndrome; $C_{a b}$ : abdominal compliance; $\mid \mathrm{AH}$ : intra-abdominal hypertension; IAP: intra-abdominal pressure; IAP ee: endexpiratory IAP; IAP ei: end-inspiratory IAP; MIAP: mean intra-abdominal pressure (geometrical mean); MIAP $P_{\mathrm{ri}}$ : respiratory-integrated mean intraabdominal pressure; $P_{\text {alv }}$ : alveolar pressure; $P_{\text {mean: }}$ mean airway pressure; $P_{\text {plat: }}$ plateau airway pressure; PEEP: positive end-expiratory pressure; TAI: thoracoabdominal index of transmission; TV: tidal volume; WSACS: World Society of the Abdominal Compartment Syndrome.
\end{abstract}

\section{Acknowledgements}

This article has been published as part of Annals of Intensive Care Volume 2 Supplement 1, 2012: Diagnosis and management of intra-abdominal hypertension and abdominal compartment syndrome. The full contents of the supplement are available online at http://www.annalsofintensivecare. com/supplements/2/S1.

\section{Author details}

${ }^{1}$ Saadat Abad Veterinary Specialty Clinic, Saadat Abad, Tehran, Iran. ${ }^{2}$ Executive Committee, World Society of the Abdominal Compartment Syndrome (WSACS), Dreef 1, Lovenjoel, 3360, Belgium. ${ }^{3} \mathrm{ICU}$ and High Care Burn Unit, Department of Intensive Care, Ziekenhuis Netwerk Antwerpen (ZNA) Stuivenberg, Lange Beeldekensstraat 267, Antwerp, 2060, Belgium.

\section{Authors' contributions}

SA and MLNGM planned the study and were responsible for the design, coordination, and drafting the manuscript. SA developed the mathematical model for MIAP calculation and performed the theoretical analyses. MLNGM performed the data collection and statistical analysis for the human pilot study. Both authors read and approved the final manuscript.

\section{Authors' information}

SA is aveterinary surgeon (DVM, DVSc) and a medical research consultant in laboratory animal researches in the field of trauma, haemorrhage, critical care, and anaesthesia. MLNGM is a former president and treasurer of the World Society of the Abdominal Compartment Syndrome and is the ICU and High Care Burn Unit Director of the Department of Intensive Care in Ziekenhuis Netwerk Antwerpen Stuivenberg.

\section{Competing interests}

MLNGM is a member of the medical advisory board of Pulsion Medical Systems, Munich, Germany.

Published: 20 December 2012

\section{References}

1. Cheatham ML, White MW, Sagraves SG, Johnson JL, Block EF: Abdominal perfusion pressure: a superior parameter in the assessment of intraabdominal hypertension. J Trauma 2000, 49(4):621-626, discussion 626627.

2. Malbrain $\mathrm{ML}$, De laet IE: Intra-abdominal hypertension: evolving concepts. Clin Chest Med 2009, 30(1):45-70.

3. Ivatury RR, Cheatham ML, Malbrain ML, Sugrue M: Abdominal Compartment Syndrome Georgetown: Landes Bioscience; 2006.

4. Malbrain ML, Cheatham ML: Definitions and pathophysiological implications of intra-abdominal hypertension and abdominal compartment syndrome. Am Surg 2011, 77(Suppl 1):S6-11.

5. Malbrain ML, Chiumello D, Pelosi P, Bihari D, Innes R, Ranieri VM, Del Turco M, Wilmer A, Brienza N, Malcangi V, Cohen J, Japiassu A, De Keulenaer BL, Daelemans $R$, Jacquet L, Laterre PF, Frank G, de Souza P, Cesana $B$, Gattinoni $L$ : Incidence and prognosis of intraabdominal hypertension in a mixed population of critically ill patients: a multiplecenter epidemiological study. Crit Care Med 2005, 33(2):315-322.

6. Malbrain ML: Different techniques to measure intra-abdominal pressure (IAP): time for a critical re-appraisal. Intensive Care Med 2004, 30(3):357-371.

7. De Keulenaer BL, De Waele JJ, Powell B, Malbrain ML: What is normal intra-abdominal pressure and how is it affected by positioning, body mass and positive end-expiratory pressure? Intensive Care Med 2009, 35(6):969-976.

8. Hunter JD: Abdominal compartment syndrome: an under-diagnosed contributory factor to morbidity and mortality in the critically ill. Postgrad Med J 2008, 84(992):293-298.

9. Malbrain ML, Cheatham ML, Kirkpatrick A, Sugrue M, Parr M, De Waele J, Balogh Z, Leppaniemi A, Olvera C, Ivatury R, D'Amours S, Wendon J, Hillman K, Johansson K, Kolkman K, Wilmer A: Results from the International Conference of Experts on Intra-abdominal Hypertension and Abdominal Compartment Syndrome. I. Definitions. Intensive Care Med 2006, 32(11):1722-1732.

10. Sturini E, Saporito A, Sugrue M, Parr MJ, Bishop G, Braschi A: Respiratory variation of intra-abdominal pressure: indirect indicator of abdominal compliance? Intensive Care Med 2008, 34(9):1632-1637.

11. Papavramidis TS, Marinis AD, Pliakos I, Kesisoglou I, Papavramidou N: Abdominal compartment syndrome - intra-abdominal hypertension: defining, diagnosing, and managing. J Emerg Trauma Shock 2011, $4(2): 279-291$.

12. Verzilli $D$, Constantin JM, Sebbane $M$, Chanques G, Jung B, Perrigault PF, Malbrain M, Jaber S: Positive end-expiratory pressure affects the value of intra-abdominal pressure in acute lung injury/acute respiratory distress syndrome patients: a pilot study. Crit Care 2010, 14(4):R137. 
13. Malbrain ML, De laet I, Cheatham M: Consensus conference definitions and recommendations on intra-abdominal hypertension (IAH) and the abdominal compartment syndrome (ACS)-the long road to the final publications, how did we get there? Acta Clin Belg 2007, 62(Suppl 1 (1)):44-59.

14. Fischbach M, Terzic J, Laugel V, Escande B, Dangelser C, Helmstetter A: Measurement of hydrostatic intraperitoneal pressure: a useful tool for the improvement of dialysis dose prescription. Pediatr Nephrol 2003, 18(10):976-980.

15. Stewart J: Calculus: Concepts and Contexts. 3 edition. Belmont: Thomson Brooks/Cole; 2005.

16. Kanani M, Elliott M: Applied Surgical Physiology Vivas London: Greenwich Medical Media Ltd; 2004

17. Raff H, Levitzky MG: Medical Physiology: A Systems Approach New York: McGraw-Hill; 2011.

18. Klabunde RE: Cardiovascular Physiology Concepts Philadelphia: Lippincott Williams \& Wilkins; 2005.

19. Chemla D, Antony I, Zamani K, Nitenberg A: Mean aortic pressure is the geometric mean of systolic and diastolic aortic pressure in resting humans. J Appl Physiol 2005, 99(6):2278-2284.

20. Moran D, Epstein Y, Keren G, Laor A, Sherez J, Shapiro Y: Calculation of mean arterial pressure during exercise as a function of heart rate. Appl Human Sci 1995, 14(6):293-295.

21. Ahmadi-Noorbakhsh S, Malbrain MLNG: Addendum: Mathematical model for calculation of mean intra-abdominal pressure, taking into account integration of inspiratory and expiratory intra-abdominal pressure. Ann Intensive Care 2012, Supplement 2 (in press).

doi:10.1186/2110-5820-2-S1-S18

Cite this article as: Ahmadi-Noorbakhsh and Malbrain: Integration of inspiratory and expiratory intra-abdominal pressure: a novel concept looking at mean intra-abdominal pressure. Annals of Intensive Care 2012 2(Suppl 1):S18.

\section{Submit your manuscript to a SpringerOpen ${ }^{\mathcal{O}}$ journal and benefit from:}

- Convenient online submission

- Rigorous peer review

- Immediate publication on acceptance

- Open access: articles freely available online

- High visibility within the field

- Retaining the copyright to your article

Submit your next manuscript at $\gg$ springeropen.com 